\title{
Structural Flexibility of Cyclosporine A is Mediated by Amide Cis- Trans Isomerization and the Chameleonic Roles of Calcium
}

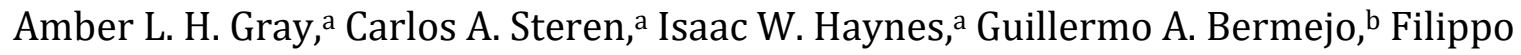 \\ Favretto, ${ }^{c}$ Markus Zweckstetter, ${ }^{\text {c,d }}$ Thanh D. Do ${ }^{\mathrm{a}, *}$ \\ a Department of Chemistry, University of Tennessee, Knoxville, TN 37996, U.S.A. \\ b Computational Biomolecular Magnetic Resonance Core, Laboratory of Chemical Physics, National Institute of \\ Diabetes and Digestive and Kidney Diseases, National Institutes of Health, Bethesda, MD 20892-0520, U.S.A. \\ c Translational Structural Biology in Dementia, German Center for Neurodegenerative Diseases (DZNE), Von- \\ Siebold-Str. 3a, 37075 Göttingen, Germany \\ dDepartment for NMR-based Structural Biology, Max Planck Institute for Biophysical Chemistry, Am Fassberg \\ 11, 37077 Göttingen, Germany
}

\section{SUPPORTING INFORMATION}

Table of Content

S1. Supporting Tables S3

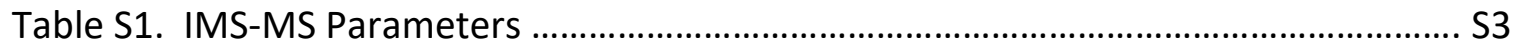

Table S2. Experimental Collisional Cross Sections.......................................................... S4

Table S3. Proton chemical shift $(\delta)$ assignments of the closed form of CycA in 90:10 $\mathrm{CD}_{3} \mathrm{OD}: \mathrm{H}_{2} \mathrm{O}$ (sample A-1), $\mathrm{CNCH}_{3}$ (sample B-1), $\mathrm{CNCH}_{3}$ (sample $\mathrm{B}-3$ ) and $\mathrm{CDCl}_{3} \ldots \ldots \ldots \ldots . . . . . \mathrm{S} 5$

Table S4. Carbon chemical shift $(\delta)$ assignments of the closed form of CycA in 90:10

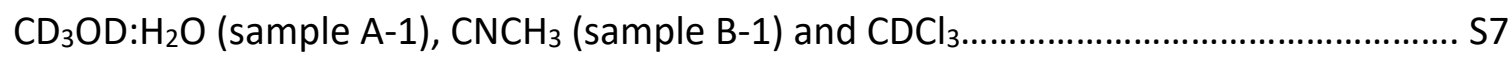

Table S5. Proton chemical shift $(\delta)$ assignments of $\mathrm{CycA}: \mathrm{Ca}^{2+}$ complex in sample $\mathrm{B}-3$ at

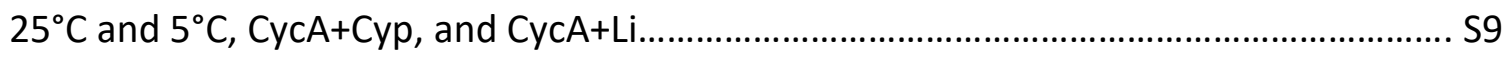

Table S6. Carbon chemical shift $(\delta)$ assignments of $\mathrm{CycA}: \mathrm{Ca}^{2+}$ complex in sample B-3 at $5^{\circ} \mathrm{C}$, CycA:Li and CycA+Cyp. S11

Table S7. ROESY peaks assignments of the closed form of CycA in sample B-1 at $25^{\circ} \mathrm{C}$ that were used for MD calculations. S13

Table S8. ROESY peaks assignments of the CycA:Ca2+ in sample B-3 at $5^{\circ} \mathrm{C}$ that were used for MD calculations. 
S2. Supporting Figures S20

Figure S1. HSQC spectrum of free CyCA in CD3OD:H2O/D2O (sample A-1). S20

Figure S2. NOE signals showing characteristics of the closed form of CycA in CD3OD:H2O/D2O. S21

Figure S3. HSQC spectrum of free CycA in CD3CN (sample B-1). S22

Figure S4. HSQC spectrum of CycA:Ca2+ in CD3CN (sample B-3). S23

Figure S5. NOESY crosspeaks showing the three main conformations in sample B-3.. S24

Figure S6. Mass spectra of CycA in methanol and acetonitrile. S25

Figure S7. Arrival time distribution spectra of CycA in methanol and acetonitrile....... S26 References Cited S27 


\section{S1. Supporting Tables}

\section{Table S1. Agilent 6560 IMS-QTOF Parameters}

The "value" column on the left is the instrumental settings used for characterization of CycA, CypA, and the CyсA-СурA mixture. The "value" column on the right is the settings used for analysis of CycA-CypA mixture in the presence of $\mathrm{Ca}^{2+}$ and $\mathrm{K}^{+}$. Parameters changed between settings are in bold.

\begin{tabular}{|c|c|c|c|}
\hline & Parameter & \multicolumn{2}{|c|}{ Value } \\
\hline \multirow{20}{*}{$\begin{array}{l}\text { Pre-IMS } \\
\text { Zone }\end{array}$} & Source: Gas Temperature & $300^{\circ} \mathrm{C}$ & $300^{\circ} \mathrm{C}$ \\
\hline & Source: Drying Gas & $5 \mathrm{~L} / \mathrm{min}$ & $5 \mathrm{~L} / \mathrm{min}$ \\
\hline & Source: Nebulizer Pressure & $13 \mathrm{psi}$ & $25 \mathrm{psi}$ \\
\hline & Source: Capillary & $3800 \mathrm{~V}$ & $3500 \mathrm{~V}$ \\
\hline & Optics I: Fragmentor & $250 \mathrm{~V}$ & $250 \mathrm{~V}$ \\
\hline & IM Front Funnel: High Pressure Funnel Delta & $110 \mathrm{~V}$ & $150 \mathrm{~V}$ \\
\hline & IM Front Funnel: High Pressure RF Delta & $180 \mathrm{~V}$ & $100 \mathrm{~V}$ \\
\hline & IM Front Funnel: Trap Funnel Delta & $160 \mathrm{~V}$ & $180 \mathrm{~V}$ \\
\hline & IM Front Funnel: Trap Funnel RF & $180 \mathrm{~V}$ & $100 \mathrm{~V}$ \\
\hline & IM Front Funnel: Trap Funnel Exit & $10 \mathrm{~V}$ & $10 \mathrm{~V}$ \\
\hline & IM Trap: Trap Entrance Grid Low & $82 \mathrm{~V}$ & $96 \mathrm{~V}$ \\
\hline & IM Trap: Trap Entrance Grid Delta & $2 \mathrm{~V}$ & $10 \mathrm{~V}$ \\
\hline & IM Trap: Trap Entance & $79 \mathrm{~V}$ & $91 \mathrm{~V}$ \\
\hline & IM Trap: Trap Exit & $76 \mathrm{~V}$ & $90 \mathrm{~V}$ \\
\hline & IM Trap: Trap Exit Grid 1 Low & $72 \mathrm{~V}$ & $87.7 \mathrm{~V}$ \\
\hline & IM Trap: Trap Exit Grid 1 Delta & $6 \mathrm{~V}$ & $4 \mathrm{~V}$ \\
\hline & IM Trap: Trap Exit Grid 2 Low & $71 \mathrm{~V}$ & $86.9 \mathrm{~V}$ \\
\hline & IM Trap: Trap Exit Grid 2 Delta & $13 \mathrm{~V}$ & $8.5 \mathrm{~V}$ \\
\hline & Acquisition: Trap Fill Time & $1000 \mu \mathrm{s}$ & $5000 \mu \mathrm{s}$ \\
\hline & Acquisition: Trap Release Time & $100 \mu \mathrm{s}$ & $150 \mu \mathrm{s}$ \\
\hline \multirow{19}{*}{$\begin{array}{l}\text { Post-IMS } \\
\text { Zone }\end{array}$} & IM Drift Tube: Drift Tube Exit & $210 \mathrm{~V}$ & $250 \mathrm{~V}$ \\
\hline & IM Rear Funnel: Rear Funnel Entance & $200 \mathrm{~V}$ & $240 \mathrm{~V}$ \\
\hline & IM Rear Funnel: Rear Funnel RF & $130 \mathrm{~V}$ & $100 \mathrm{~V}$ \\
\hline & IM Rear Funnel: Rear Funnel Exit & $35 \mathrm{~V}$ & $36 \mathrm{~V}$ \\
\hline & IM Rear Funnel: IM Hex Entrance & $42 \mathrm{~V}$ & $34 \mathrm{~V}$ \\
\hline & IM Rear Funnel: IM Hex Delta & $-8 \mathrm{~V}$ & $-8 \mathrm{~V}$ \\
\hline & Optics 1: Oct Entrance Lens & $32 \mathrm{~V}$ & $32 \mathrm{~V}$ \\
\hline & Optics 1: Lens 1 & $28.3 \mathrm{~V}$ & $28.3 \mathrm{~V}$ \\
\hline & Optics 1: Lens 2 & $15.8 \mathrm{~V}$ & $15.8 \mathrm{~V}$ \\
\hline & Quad: Quad DC & $26.6 \mathrm{~V}$ & $26.6 \mathrm{~V}$ \\
\hline & Quad: Postfilter DC & $26.5 \mathrm{~V}$ & $26.5 \mathrm{~V}$ \\
\hline & Cell: Gas Flow & 22 psi & 22 psi \\
\hline & Cell: Cell Entrance & $25.6 \mathrm{~V}$ & $25.6 \mathrm{~V}$ \\
\hline & Cell: Hex DC & $24.2 \mathrm{~V}$ & $24.2 \mathrm{~V}$ \\
\hline & Cell: Hex Delta & $-9 \mathrm{~V}$ & $-9 \mathrm{~V}$ \\
\hline & Cell: Hex2 DC & $15 \mathrm{~V}$ & $15 \mathrm{~V}$ \\
\hline & Cell: Hex2 DV & $-3 V$ & $-3 \mathrm{~V}$ \\
\hline & Optics 2: Hex3 DC & $11.8 \mathrm{~V}$ & $11.8 \mathrm{~V}$ \\
\hline & Extractor: Ion Focus & $5.6 \mathrm{~V}$ & $5.6 \mathrm{~V}$ \\
\hline
\end{tabular}


Table S2. Experimental Collisional Cross Sections

\begin{tabular}{|c|c|}
\hline Species & $\begin{array}{c}\text { Experimental CCS } \\
\left(\AA^{2}\right)\end{array}$ \\
\hline \multicolumn{2}{|l|}{ Cyclosporin A } \\
\hline$\left[\mathrm{CycA}+\mathrm{H}^{+}\right]_{\text {closed }}^{+1}$ & 281.7 \\
\hline$\left[\mathrm{CycA}+\mathrm{H}^{+}\right]_{\text {bent }}^{+1}$ & 271.1 \\
\hline$\left[\mathrm{CycA}+2 \mathrm{H}^{+}\right]_{\text {bent }}^{+2}$ & 297.2 \\
\hline$\left[\mathrm{CycA}+\mathrm{Ca}^{2+}\right]_{\text {open }}^{+2}$ & 296.8 \\
\hline$\left[\mathrm{CycA}+\mathrm{Ca}^{2+}\right]_{\text {closed }}^{+2}$ & 280.4 \\
\hline$\left[\mathrm{CycA}+\mathrm{Ca}^{2+}\right]_{\text {bent }}^{+2}$ & 269.8 \\
\hline$\left[2 \mathrm{CycA}+\mathrm{Ca}^{2+}\right]^{+2}$ & 444.4 \\
\hline$\left[\mathrm{CycA}+\mathrm{Na}^{+}\right]^{+1}$ & 267.2 \\
\hline \multicolumn{2}{|c|}{ Cyclosporin A-Cyclophilin A Complex } \\
\hline$\left[\mathrm{CycA}+\mathrm{CypA}+8 \mathrm{H}^{+}\right]^{+8}$ & 1726.3 \\
\hline$\left[\mathrm{CycA}+\mathrm{CypA}+9 \mathrm{H}^{+}\right]^{+9}$ & 1792.4 \\
\hline$\left[\mathrm{CycA}+\mathrm{CypA}+8 \mathrm{H}^{+}\right]^{+8}\left(\right.$ Presence of $\left.\mathrm{Ca}^{2+}\right)$ & 1774.4 \\
\hline$\left[\mathrm{CycA}+\mathrm{CypA}+9 \mathrm{H}^{+}\right]^{+9}\left(\right.$ Presence of $\left.\mathrm{Ca}^{2+}\right)$ & 1842.4 \\
\hline$\left[\text { CycA }+ \text { CypA }+8 \mathrm{H}^{+}\right]^{+8}\left(\right.$ Presence of $\left.\mathrm{K}^{+}\right)$ & 1767.7 \\
\hline$\left[\mathrm{CycA}+\mathrm{CypA}+9 \mathrm{H}^{+}\right]^{+9}\left(\right.$ Presence of $\left.\mathrm{K}^{+}\right)$ & 1837.9 \\
\hline \multicolumn{2}{|l|}{ Cyclophilin A } \\
\hline$[\text { CypA }]^{+7}$ & 1508.0 \\
\hline$[\text { CypA }]^{+8}$ & 1588.2 \\
\hline$[\text { CурA }]^{+9}$ & 2259.0 \\
\hline$[\text { СурA }]^{+10}$ & 2427.1 \\
\hline$[\text { СурA }]^{+11}$ & 2818.7 \\
\hline$[\text { СурA }]^{+12}$ & 2983.9 \\
\hline$[\text { СурA }]^{+13}$ & 3085.4 \\
\hline$[\text { СурA }]^{+14}$ & 3196.9 \\
\hline$[\text { СурA }]^{+15}$ & 3295.4 \\
\hline$[\text { CypA }]^{+16}$ & 3390.8 \\
\hline$[\text { CypA }]^{+17}$ & 3473.9 \\
\hline$[\text { СурA }]^{+18}$ & 3586.0 \\
\hline$[\text { СурА }]^{+19}$ & 3725.4 \\
\hline$[\text { СурA }]^{+20}$ & 3749.5 \\
\hline$[\text { CypA }]^{+21}$ & 3897.9 \\
\hline$[\text { CypA }]^{+8}\left(\right.$ Presence of $\left.\mathrm{K}^{+}\right)$ & 1679.2 \\
\hline$[\mathrm{CypA}]^{+8}\left(\right.$ Presence of $\left.\mathrm{Ca}^{2+}\right)$ & 1698.1 \\
\hline
\end{tabular}

The CCS values reported for cyclosporine $A$ are in water with minor amount of methanol ( 1\%). The CycA-CypA mixture are in $20 \mathrm{mM}$ ammonium acetate, or $200 \mathrm{mM}$ ammonium acetate for the complexes in the presence of $\mathrm{Ca}^{2+} / \mathrm{K}^{+}$. The CCS for cyclophilin A is in water, except for $z=+7$ through +9 which are in $20 \mathrm{mM}$ ammonium acetate.

Due to the low intensity of the mass spectral peaks, there may be a small variance $(\sim 2 \%)$ in the reported CCS for low-charge state CypA ions $(z=+7$ and $z=+8)$ and CycA:CypA complexes. 
Table S3. Proton chemical shift ( $\delta$ ) assignments of the closed form of $C y c A$ in 90:10 $\mathrm{CD}_{3} \mathrm{OD}: \mathrm{H}_{2} \mathrm{O}$ (sample A-1), $\mathrm{CNCH}_{3}$ (sample B-1), $\mathrm{CNCH}_{3}$ (sample B-3) and $\mathrm{CDCl}_{3}$.

\begin{tabular}{|c|c|c|c|c|c|}
\hline Residue & Proton & $\begin{array}{c}\mathrm{CD}_{3} \mathrm{OD} @ 25^{\circ} \mathrm{C} \\
\delta \text { [ppm] } \\
\end{array}$ & $\begin{array}{c}\mathrm{CNCH}_{3} \text { (B1) @ } 25^{\circ} \mathrm{C} \\
\delta[\mathrm{ppm}] \\
\end{array}$ & $\begin{array}{c}\mathrm{CNCH}_{3} \text { (B3) @ } 25^{\circ} \mathrm{C} \\
\delta[\mathrm{ppm}] \\
\end{array}$ & $\begin{array}{c}\mathrm{CDCl}_{3} @ \sim 23^{\circ} \mathrm{C}^{1} \\
\delta[\mathrm{ppm}] \\
\end{array}$ \\
\hline \multirow[t]{10}{*}{$\begin{array}{c}1 \\
\text { MeBmt }\end{array}$} & $\mathrm{MeN}$ & $3.42^{\mathrm{a}}$ & 3.41 & 3.42 & 3.52 \\
\hline & $\mathrm{H} \alpha$ & 5.33 & 5.33 & 5.34 & 5.45 \\
\hline & $H \beta$ & 3.91 & 3.83 & 3.84 & 3.82 \\
\hline & $\mathrm{H} \delta 1$ & 0.82 & 0.74 & 0.74 & 0.72 \\
\hline & $\mathrm{H} \delta 21$ & 1.76 & 1.72 & 1.75 & 1.73 \\
\hline & $\mathrm{H} \delta 22$ & 2.20 & 2.26 & 2.27 & 2.41 \\
\hline & $\mathrm{H} \varepsilon$ & 5.31 & 5.34 & 5.35 & 5.36 \\
\hline & $\mathrm{H} \gamma 2$ & 1.59 & 1.57 & 1.56 & 1.63 \\
\hline & $\mathrm{H \eta}$ & 1.60 & 1.60 & 1.61 & 1.62 \\
\hline & $\mathrm{H} \zeta$ & 5.31 & 5.34 & 5.34 & 5.35 \\
\hline \multirow[t]{5}{*}{$2 \mathrm{Abu}$} & $\mathrm{HN}$ & $8.15^{a}$ & 7.71 & 7.63 & 7.93 \\
\hline & $\mathrm{H} \alpha$ & 4.98 & 4.98 & 4.98 & 5.03 \\
\hline & $H \beta 2$ & 1.66 & 1.69 & 1.70 & 1.74 \\
\hline & $H \beta 3$ & 1.75 & 1.60 & 1.61 & 1.60 \\
\hline & $\mathrm{H} \gamma$ & 0.88 & 0.84 & 0.85 & 0.87 \\
\hline \multirow[t]{3}{*}{3 Sar } & MeN & $3.38^{a}$ & 3.32 & 3.31 & 3.40 \\
\hline & $\mathrm{H} \alpha 2$ & 4.73 & 4.68 & 4.73 & 4.76 \\
\hline & $\mathrm{H} \alpha 3$ & 3.42 & 3.30 & 3.34 & 3.23 \\
\hline \multirow[t]{7}{*}{$\begin{array}{c}4 \\
\text { MeLeu }\end{array}$} & $\mathrm{MeN}$ & 3.04 & 3.06 & 3.05 & 3.11 \\
\hline & $\mathrm{H} \alpha$ & 5.40 & 5.29 & 5.28 & 5.34 \\
\hline & $H \beta 2$ & 2.38 & 1.86 & 1.86 & 2.00 \\
\hline & $H \beta 3$ & 1.70 & 1.64 & 1.65 & 1.64 \\
\hline & $\mathrm{H} \delta 1$ & 0.88 & 0.94 & - & 0.95 \\
\hline & $\mathrm{H} \delta 2$ & 0.97 & 0.85 & 0.86 & 0.88 \\
\hline & $\mathrm{H} \gamma$ & 1.48 & 1.49 & 1.49 & 1.44 \\
\hline \multirow[t]{5}{*}{$5 \mathrm{Val}$} & $\mathrm{HN}$ & 7.84 & 7.49 & 7.44 & 7.47 \\
\hline & $\mathrm{H} \alpha$ & 4.71 & 4.61 & 4.62 & 4.67 \\
\hline & $H \beta$ & 2.45 & 2.42 & 2.44 & 2.41 \\
\hline & $\mathrm{H} \gamma 1$ & 1.05 & 1.04 & 1.04 & 1.06 \\
\hline & $\mathrm{H} \gamma 2$ & 0.88 & 0.85 & 0.87 & 0.90 \\
\hline \multirow[t]{4}{*}{$\begin{array}{c}6 \\
\text { MeLeu }\end{array}$} & $\mathrm{MeN}$ & $3.20^{\mathrm{a}}$ & 3.17 & 3.17 & 3.25 \\
\hline & $\mathrm{H} \alpha$ & 5.17 & 5.03 & 5.07 & 5.02 \\
\hline & $H \beta 2$ & 2.03 & 1.99 & 1.94 & 2.06 \\
\hline & $H \beta 3$ & 1.38 & 1.36 & 1.40 & 1.41 \\
\hline
\end{tabular}




\begin{tabular}{|c|c|c|c|c|c|}
\hline & $\mathrm{H} \delta 1$ & 0.85 & 0.83 & 0.85 & 0.85 \\
\hline & $\mathrm{H} \delta 2$ & 0.93 & 0.90 & 0.90 & 0.94 \\
\hline & $\mathrm{H} \gamma$ & 1.75 & 1.70 & 1.67 & 1.76 \\
\hline \multirow[t]{3}{*}{7 Ala } & $\mathrm{HN}$ & 7.89 & 7.52 & 7.59 & 7.75 \\
\hline & $\mathrm{H} \alpha$ & 4.41 & 4.32 & 4.33 & 4.52 \\
\hline & $\mathrm{H} \beta \#$ & 1.36 & 1.31 & 1.32 & 1.36 \\
\hline \multirow[t]{3}{*}{8 D-Ala } & $\mathrm{HN}$ & 7.15 & 7.20 & 7.22 & 7.18 \\
\hline & $\mathrm{H} \alpha$ & 4.84 & 4.77 & 4.78 & 4.84 \\
\hline & $\mathrm{H} \beta$ & 1.28 & 1.20 & 1.21 & 1.26 \\
\hline \multirow[t]{7}{*}{$\begin{array}{c}9 \\
\text { MeLeu }\end{array}$} & MeN & $3.16^{a}$ & 3.08 & 3.08 & 3.12 \\
\hline & $\mathrm{H} \alpha$ & 5.73 & 5.66 & 5.66 & 5.70 \\
\hline & $H \beta 2$ & 2.08 & 1.24 & 1.264 & 1.25 \\
\hline & $\mathrm{H} \beta 3$ & 1.26 & 2.05 & 2.029 & 2.13 \\
\hline & $\mathrm{H} \delta 1$ & 0.973 & 0.95 & 0.95 & 0.97 \\
\hline & $\mathrm{H} \delta 2$ & 0.895 & 0.88 & 0.88 & 0.89 \\
\hline & $\mathrm{H} \gamma$ & 1.39 & 1.37 & 1.37 & 1.32 \\
\hline \multirow[t]{7}{*}{$\begin{array}{c}10 \\
\text { MeLeu }\end{array}$} & MeN & $2.71^{a}$ & 2.64 & 2.65 & 2.70 \\
\hline & $\mathrm{H} \alpha$ & 5.16 & 5.04 & 5.03 & 5.10 \\
\hline & $\mathrm{H} \beta 2$ & 2.02 & 2.11 & 2.13 & 2.13 \\
\hline & $\mathrm{H} \beta 3$ & 1.20 & 1.19 & 1.48 & 1.24 \\
\hline & $\mathrm{H} \delta 1$ & 1.01 & 1.04 & 1.04 & 0.98 \\
\hline & $\mathrm{H} \delta 2$ & 1.05 & 1.00 & 1.00 & 0.98 \\
\hline & $\mathrm{H} \gamma$ & 1.51 & 1.48 & 1.19 & 1.49 \\
\hline \multirow[t]{5}{*}{$\begin{array}{c}11 \\
\text { MeVal }\end{array}$} & $\mathrm{MeN}$ & $2.76^{a}$ & 2.69 & 2.73 & 2.71 \\
\hline & $\mathrm{H} \alpha$ & 5.22 & 5.15 & 5.17 & 5.15 \\
\hline & $H \beta$ & 2.21 & 2.12 & 2.18 & 2.17 \\
\hline & $\mathrm{H} \gamma 1$ & 0.97 & 0.84 & 0.95 & 1.01 \\
\hline & $\mathrm{H} \gamma 2$ & 0.87 & 0.94 & 0.84 & 0.87 \\
\hline
\end{tabular}

${ }^{1}$ Chemical shifts from Kesslet et al. (ref. 1)

${ }^{\text {a }}$ Chemical shifts obtained at $15^{\circ} \mathrm{C}$ 
Table S4. Carbon chemical shift $(\delta)$ assignments of the closed form of CycA in 90:10 $\mathrm{CD}_{3} \mathrm{OD}: \mathrm{H}_{2} \mathrm{O}$ (sample A-1), $\mathrm{CNCH}_{3}$ (sample B-1) and $\mathrm{CDCl}_{3}$.

\begin{tabular}{|c|c|c|c|c|}
\hline \multirow[t]{2}{*}{ Residue } & \multirow[t]{2}{*}{ Carbon } & \multirow{2}{*}{$\begin{array}{c}\mathrm{CD}_{3} \mathrm{OD} @ 25^{\circ} \mathrm{C} \\
\delta \text { [ppm] }\end{array}$} & \multirow{2}{*}{$\begin{array}{c}\mathrm{CNCH}_{3}(\mathrm{~B} 1) @ 25^{\circ} \mathrm{C} \\
\delta[\mathrm{ppm}]\end{array}$} & \multirow{2}{*}{$\begin{array}{c}\mathrm{CDCl}_{3} @ 23^{\circ} \mathrm{C}^{2} \\
\delta[\mathrm{ppm}]\end{array}$} \\
\hline & & & & \\
\hline \multirow[t]{10}{*}{$1 \mathrm{MeBmt}$} & NMe & 33.9 & - & 35.3 \\
\hline & $\mathrm{C} \alpha$ & 60.0 & 59.5 & - \\
\hline & $C \beta$ & 74.1 & 74.5 & 76.8 \\
\hline & $\mathrm{c} \delta 1$ & 17.8 & 17.4 & 15.6 \\
\hline & c 821 & 35.5 & 35.8 & 36.7 \\
\hline & c $\delta 22$ & - & - & 36.7 \\
\hline & $\mathrm{C} \varepsilon$ & 131.2 & 130.9 & 130.0 \\
\hline & $\mathrm{C} \gamma$ & 36.2 & 36.3 & 36.7 \\
\hline & $\mathrm{Cn}$ & 18.2 & 18.1 & 18.2 \\
\hline & $C \zeta$ & 127.0 & 126.7 & 127.5 \\
\hline \multirow[t]{4}{*}{$2 \mathrm{Abu}$} & $\mathrm{C} \alpha$ & 50.4 & 49.4 & 53.0 \\
\hline & $C \beta 2$ & 26.3 & 25.8 & 24.3 \\
\hline & $C \beta 3$ & - & - & 24.3 \\
\hline & $\mathrm{C} \gamma$ & 10.4 & 10.2 & 10.9 \\
\hline \multirow[t]{3}{*}{3 Sar } & NMe & - & 39.4 & 36.9 \\
\hline & $\mathrm{C} \alpha 2$ & 39.4 & 39.4 & 50.9 \\
\hline & $\mathrm{C} \alpha 3$ & 51.2 & - & 51.0 \\
\hline \multirow[t]{7}{*}{4 MeLeu } & NMe & 31.8 & 31.8 & 29.6 \\
\hline & $\mathrm{C} \alpha$ & 56.6 & 56.0 & 54.3 \\
\hline & $C \beta 2$ & - & 36.6 & 36.6 \\
\hline & $C \beta 3$ & - & - & 36.6 \\
\hline & $\mathrm{C} \delta 1$ & - & - & 23.2 \\
\hline & $\mathrm{C} \delta 2$ & - & 21.4 & 22.3 \\
\hline & $\mathrm{C} \gamma$ & - & - & 25.5 \\
\hline \multirow[t]{4}{*}{$5 \mathrm{Val}$} & $\mathrm{C} \alpha$ & 56.5 & 55.9 & 55.1 \\
\hline & $C \beta$ & 32.5 & 31.8 & 30.0 \\
\hline & $C_{\gamma 1}$ & 20.0 & 19.9 & 21.1 \\
\hline & $C_{\gamma} 2$ & 18.8 & 18.5 & 18.3 \\
\hline \multirow[t]{7}{*}{6 MeLeu } & $\mathrm{NMe}$ & 32.1 & 32.0 & 31.3 \\
\hline & $\mathrm{C} \alpha$ & 57.7 & 55.9 & 55.3 \\
\hline & $C \beta 2$ & 38.0 & 37.8 & - \\
\hline & $C \beta 3$ & - & - & - \\
\hline & $\mathrm{C} \delta 1$ & 22.6 & 24.3 & 24.2 \\
\hline & $\mathrm{C} \delta 2$ & 24.4 & 22.4 & 21.0 \\
\hline & $\mathrm{C} \gamma$ & 26.3 & 26.1 & 25.0 \\
\hline $7 \mathrm{Ala}$ & $\mathrm{C} \alpha$ & 49.9 & 49.6 & 51.4 \\
\hline
\end{tabular}




\begin{tabular}{|c|c|c|c|c|}
\hline & $C \beta$ & 16.4 & 16.5 & 16.6 \\
\hline \multirow[t]{2}{*}{8 D-Ala } & $\mathrm{C} \alpha$ & 46.7 & 45.9 & 46.6 \\
\hline & $C \beta$ & 17.9 & 18.4 & 15.8 \\
\hline \multirow[t]{7}{*}{9 MeLeu } & NMe & 30.2 & 30.0 & 30.8 \\
\hline & $\mathrm{C} \alpha$ & 49.8 & 49.2 & 54.5 \\
\hline & $C \beta 2$ & 40.0 & 39.5 & 38.5 \\
\hline & $C \beta 3$ & - & - & 38.5 \\
\hline & $\mathrm{C} \delta 1$ & 18.4 & 23.7 & 24.4 \\
\hline & $C \delta 2$ & 22.3 & 22.1 & 23.6 \\
\hline & $\mathrm{C} \gamma$ & 25.7 & - & 24.6 \\
\hline \multirow[t]{7}{*}{10 MeLeu } & NMe & 30.7 & 30.1 & 32.9 \\
\hline & $\mathrm{C} \alpha$ & 58.6 & 58.1 & 53.3 \\
\hline & $C \beta 2$ & 41.8 & 41.5 & 37.8 \\
\hline & $C \beta 3$ & - & - & 37.8 \\
\hline & $\mathrm{C} \delta 1$ & 24.3 & 23.4 & 24.2 \\
\hline & $\mathrm{C} \delta 2$ & 23.8 & 24.1 & 20.8 \\
\hline & $\mathrm{C} \gamma$ & 25.9 & - & 25.6 \\
\hline \multirow[t]{5}{*}{$11 \mathrm{MeV} a \mathrm{l}$} & NMe & 31.2 & 30.5 & 30.4 \\
\hline & $\mathrm{C} \alpha$ & 59.2 & 58.4 & 59.4 \\
\hline & $C \beta$ & 30.3 & 29.7 & 27.0 \\
\hline & $C_{\gamma 1}$ & 19.2 & 18.9 & 18.3 \\
\hline & $C \gamma 2$ & 20.3 & 20.1 & 20.4 \\
\hline
\end{tabular}

${ }^{2}$ Chemical shifts from Köck et al. (ref. 2) 
Table S5. Proton chemical shift $(\delta)$ assignments of $\mathrm{CycA}: \mathrm{Ca}^{2+}$ complex in sample $\mathrm{B}-3$ at $25^{\circ} \mathrm{C}$ and $5^{\circ} \mathrm{C}$, CycA+Cyp, and CycA+Li.

\begin{tabular}{|c|c|c|c|c|c|}
\hline Residue & Atom & $\begin{array}{c}\text { CycA:Ca }{ }^{2+}(\mathrm{B} 3) @ 25^{\circ} \mathrm{C} \\
\delta[\mathrm{ppm}]\end{array}$ & 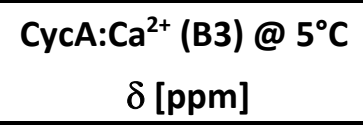 & $\begin{array}{c}\text { СycA+CypA @ 20 }{ }^{\circ} \mathrm{C}^{2} \\
\delta[\mathrm{ppm}] \\
\end{array}$ & $\begin{array}{c}\text { CycA+Li @ 27 } 7^{\circ} \mathrm{C}^{2} \\
\delta[\mathrm{ppm}] \\
\end{array}$ \\
\hline \multirow[t]{10}{*}{$1 \mathrm{MeBmt}$} & MeN & 2.99 & 2.96 & 3.28 & 3.10 \\
\hline & $\mathrm{H} \alpha$ & 5.47 & 5.41 & 6.82 & 5.50 \\
\hline & $H \beta$ & 4.08 & 4.07 & 4.04 & 4.71 \\
\hline & $\mathrm{H} \delta 1$ & 0.84 & 0.82 & - & 0.83 \\
\hline & $\mathrm{H} \delta 21$ & 2.01 & 1.99 & 2.25 & 1.83 \\
\hline & $\mathrm{H} \delta 22$ & 2.47 & 2.48 & 2.60 & - \\
\hline & $\mathrm{H} \varepsilon$ & 5.50 & 5.48 & 5.46 & 5.6 \\
\hline & $\mathrm{H} \gamma 2$ & 1.50 & 1.47 & 1.50 & 1.55 \\
\hline & $\mathrm{H \eta}$ & 1.67 & 1.66 & 1.61 & 1.60 \\
\hline & $\mathrm{H} \zeta$ & 5.50 & 5.48 & 6.15 & 5.40 \\
\hline \multirow[t]{5}{*}{$2 \mathrm{Abu}$} & $\mathrm{HN}$ & 6.73 & 6.75 & 6.42 & 9.30 \\
\hline & $\mathrm{H} \alpha$ & 4.82 & 4.78 & 5.02 & 4.44 \\
\hline & $H \beta 1$ & 1.82 & 1.81 & 1.65 & 2.48 \\
\hline & $H \beta 3$ & 1.65 & 1.63 & 1.45 & 1.83 \\
\hline & $\mathrm{H} \gamma \#$ & 1.05 & 1.05 & 0.67 & 1.08 \\
\hline \multirow[t]{3}{*}{3 Sar } & $\mathrm{MeN}$ & 3.11 & 3.12 & 2.93 & 3.17 \\
\hline & $\mathrm{H} \alpha 2$ & 4.82 & 4.79 & 5.31 & 5.05 \\
\hline & $\mathrm{H} \alpha 3$ & 3.52 & 3.50 & 3.04 & 4.23 \\
\hline \multirow[t]{7}{*}{4 MeLeu } & MeN & 2.83 & 2.81 & 2.84 & 3.14 \\
\hline & $\mathrm{H} \alpha$ & 5.03 & 5.00 & 5.15 & 5.46 \\
\hline & $H \beta 2$ & 1.82 & 1.85 & 1.68 & 1.89 \\
\hline & $H \beta 3$ & 1.19 & 1.63 & 1.57 & 1.35 \\
\hline & $\mathrm{H} \delta 1$ & 0.93 & 0.94 & 0.93 & 0.97 \\
\hline & $\mathrm{H} \delta 2$ & 0.85 & 0.90 & 0.88 & 0.90 \\
\hline & $\mathrm{H} \gamma$ & 1.43 & 1.40 & 1.38 & 1.39 \\
\hline \multirow[t]{5}{*}{$5 \mathrm{Val}$} & $\mathrm{HN}$ & 6.48 & 6.66 & 8.53 & 9.06 \\
\hline & $\mathrm{H} \alpha$ & 4.59 & 4.56 & 4.30 & 4.93 \\
\hline & $H \beta$ & 2.38 & 2.42 & 2.02 & 1.84 \\
\hline & $\mathrm{H} \gamma 1$ & 0.83 & 0.89 & 0.94 & 1.09 \\
\hline & $\mathrm{H} \gamma 2$ & 0.82 & 0.72 & 0.93 & 0.89 \\
\hline \multirow[t]{6}{*}{6 MeLeu } & MeN & 2.77 & 2.70 & 3.09 & 3.23 \\
\hline & $\mathrm{H} \alpha$ & 5.28 & 5.27 & 5.42 & 5.45 \\
\hline & $H \beta 2$ & 2.37 & 2.40 & 1.82 & 1.95 \\
\hline & $\mathrm{H} \beta 3$ & 1.28 & 1.19 & 1.73 & 1.73 \\
\hline & $\mathrm{H} \delta 1$ & 0.90 & 0.88 & 0.89 & 0.87 \\
\hline & $\mathrm{H} \delta 2$ & 0.94 & 0.92 & 0.78 & 0.87 \\
\hline
\end{tabular}




\begin{tabular}{|c|c|c|c|c|c|}
\hline & $\mathrm{H} \gamma$ & 1.20 & 1.18 & 1.60 & 1.22 \\
\hline \multirow[t]{3}{*}{7 Ala } & $\mathrm{HN}$ & 6.94 & 7.08 & 7.56 & 6.32 \\
\hline & $\mathrm{H} \alpha$ & 4.58 & 4.53 & 4.20 & 4.89 \\
\hline & $H \beta$ & 1.29 & 1.30 & 1.39 & 1.26 \\
\hline \multirow[t]{3}{*}{8 D-Ala } & $\mathrm{HN}$ & 9.85 & 9.87 & 8.33 & 9.33 \\
\hline & $\mathrm{H} \alpha$ & 4.73 & 4.71 & 4.68 & 4.81 \\
\hline & $H \beta$ & 1.40 & 1.38 & 1.16 & 1.39 \\
\hline \multirow[t]{7}{*}{9 MeLeu } & MeN & 2.91 & 2.89 & 2.61 & 2.93 \\
\hline & $\mathrm{H} \alpha$ & 5.72 & 5.72 & 5.19 & 5.22 \\
\hline & $H \beta 2$ & 1.62 & 1.40 & 1.13 & 1.98 \\
\hline & $H \beta 3$ & 1.58 & 1.71 & -0.15 & 1.22 \\
\hline & $\mathrm{H} \delta 1$ & 0.98 & 0.94 & 0.54 & 0.86 \\
\hline & $\mathrm{H} \delta 2$ & 0.96 & 1.00 & 0.32 & 0.86 \\
\hline & $\mathrm{H} \gamma$ & 1.36 & 1.32 & 0.77 & 1.49 \\
\hline \multirow[t]{7}{*}{10 MeLeu } & MeN & 3.03 & 3.02 & 2.83 & 2.93 \\
\hline & $\mathrm{H} \alpha$ & 5.36 & 5.34 & 5.98 & 5.68 \\
\hline & $H \beta 2$ & 2.79 & 2.32 & 1.55 & 1.92 \\
\hline & $H \beta 3$ & 1.21 & 1.18 & 1.47 & 1.42 \\
\hline & $\mathrm{H} \delta 1$ & 0.96 & 0.96 & 1.10 & 1.07 \\
\hline & $\mathrm{H} \delta 2$ & 0.91 & 0.90 & 1.10 & 0.95 \\
\hline & $\mathrm{H} \gamma$ & 1.71 & 1.71 & 1.45 & 1.61 \\
\hline \multirow[t]{5}{*}{$11 \mathrm{MeVal}$} & MeN & 2.86 & 2.83 & 2.73 & 2.99 \\
\hline & $\mathrm{H} \alpha$ & 4.99 & 4.95 & 4.55 & 5.59 \\
\hline & $H \beta$ & 2.29 & 2.28 & 1.47 & 2.29 \\
\hline & $\mathrm{H} \gamma 1$ & 0.88 & 0.86 & 0.47 & 0.99 \\
\hline & $\mathrm{H} \gamma 2$ & 0.75 & 0.72 & -0.69 & 0.66 \\
\hline
\end{tabular}

\footnotetext{
${ }^{2}$ Chemical shifts from Köck et al. (ref. 2)
} 


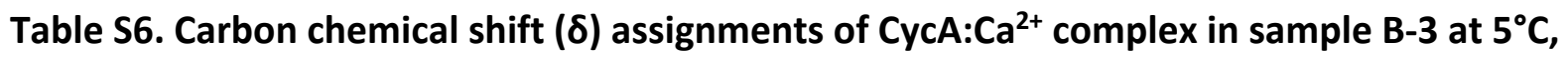
CycA:Li and CycA+Cyp.

\begin{tabular}{|c|c|c|c|c|}
\hline Residue & Atom & $\begin{array}{c}\text { CycA:Ca2+ (B3) @ 5C } \\
\delta[p p m]\end{array}$ & $\begin{array}{c}\text { СусA+CypA }{ }^{2} \\
\delta[p p m]\end{array}$ & $\begin{array}{l}\text { CycA+Li }{ }^{2} \\
\delta[p p m]\end{array}$ \\
\hline \multirow[t]{9}{*}{$1 \mathrm{MeBmt}$} & $\mathrm{C} \alpha$ & 58.2 & 58.1 & 60.7 \\
\hline & $C \beta$ & 76.8 & 75.7 & 75.0 \\
\hline & $\mathrm{C} \delta 1$ & 15.6 & - & - \\
\hline & $C \delta 2$ & 36.7 & 34.0 & 37.2 \\
\hline & $\mathrm{C} \varepsilon$ & 130.0 & 124.3 & 132.4 \\
\hline & $C \gamma 2$ & 36.8 & 25.0 & 38.0 \\
\hline & $\mathrm{Cn}$ & 18.2 & 17.2 & 18.3 \\
\hline & $c \zeta$ & 127.5 & 130.1 & 125.8 \\
\hline & $\mathrm{CN}$ & 35.3 & 34.4 & 36.5 \\
\hline \multirow[t]{3}{*}{$2 \mathrm{Abu}$} & $\mathrm{C} \alpha$ & 53.0 & 50.7 & 55.7 \\
\hline & $C \beta$ & 24.3 & 24.4 & 23.1 \\
\hline & $\mathrm{C} \gamma$ & 10.9 & 9.8 & 12.0 \\
\hline \multirow[t]{2}{*}{3 Sar } & $\mathrm{C} \alpha$ & 51.0 & 50.9 & 51.3 \\
\hline & $\mathrm{CN}$ & 36.9 & 33.7 & 36.7 \\
\hline \multirow[t]{7}{*}{4 MeLeu } & $\mathrm{C} \alpha$ & 54.3 & 53.8 & 54.8 \\
\hline & $C \beta$ & 36.6 & 36.0 & 39.3 \\
\hline & $C \delta 1$ & 24.2 & 21.8 & 23.8 \\
\hline & $\mathrm{C} \delta 2$ & & 21.5 & 22.7 \\
\hline & $\mathrm{C} \gamma$ & 25.5 & 23.8 & 25.7 \\
\hline & $\mathrm{C} \gamma$ & - & - & - \\
\hline & $\mathrm{CN}$ & 29.6 & 29.3 & 30.7 \\
\hline \multirow[t]{4}{*}{$5 \mathrm{Val}$} & $\mathrm{C} \alpha$ & 55.1 & 56.4 & 55.6 \\
\hline & $C \beta$ & 30.0 & 29.4 & 31.7 \\
\hline & $C \gamma 1$ & 19.8 & 18.2 & 20.5 \\
\hline & $\mathrm{C} \gamma 2$ & - & 19.4 & 18.7 \\
\hline \multirow[t]{6}{*}{6 MeLeu } & $\mathrm{C} \alpha$ & 55.3 & 54.8 & 57.5 \\
\hline & $\mathrm{C} \beta$ & & 37.0 & 40.4 \\
\hline & $C \delta 1$ & 24.2 & 22.4 & 23.6 \\
\hline & $\mathrm{C} \delta 2$ & - & 19.8 & 21.7 \\
\hline & $\mathrm{C} \gamma$ & 25.0 & 24.0 & 25.2 \\
\hline & $\mathrm{CN}$ & 31.3 & 31.8 & 33.4 \\
\hline \multirow[t]{2}{*}{7 Ala } & $\mathrm{C} \alpha$ & 51.4 & 49.3 & 49.5 \\
\hline & $C \beta$ & 16.6 & 17.0 & 18.0 \\
\hline 8 D-Ala & $\mathrm{C} \alpha$ & 46.6 & 45.3 & 46.2 \\
\hline
\end{tabular}




\begin{tabular}{lcccc} 
& $C \beta$ & 15.8 & 15.5 & 16.0 \\
\hline 9 MeLeu & $\mathrm{C} \alpha$ & 54.5 & 52.1 & 54.6 \\
& $\mathrm{C} \beta$ & 38.5 & 36.4 & 39.0 \\
& $\mathrm{C} \gamma$ & 24.6 & 22.6 & 25.9 \\
& $\mathrm{C} \delta 1$ & - & 23.2 & 22.9 \\
& $\mathrm{C} \delta 2$ & - & 20.2 & 21.4 \\
& $\mathrm{CN}$ & 30.8 & 29.2 & 30.7 \\
\hline 10 MeLeu & $\mathrm{C} \alpha$ & 53.3 & 52.9 & 50.8 \\
& $\mathrm{C} \beta$ & 37.8 & 36.9 & 38.8 \\
& $\mathrm{C} \gamma$ & 25.6 & 25.0 & 26.3 \\
& $\mathrm{C} \delta 1$ & - & 23.5 & 23.8 \\
& $\mathrm{C} \delta 2$ & - & 19.1 & 21.6 \\
& $\mathrm{CN}$ & 32.9 & 32.2 & 31.7 \\
\hline $11 \mathrm{MeVal}$ & $\mathrm{C} \alpha$ & 59.4 & 59.3 & 60.5 \\
& $\mathrm{C} \beta$ & 27.0 & 24.7 & 27.8 \\
& $\mathrm{C} \gamma 1$ & 20.4 & 18.8 & 21.0 \\
& $\mathrm{C} \gamma 2$ & 18.3 & 16.3 & 18.0 \\
& $\mathrm{CN}$ & 30.4 & 33.2 & 31.2 \\
\hline
\end{tabular}

${ }^{2}$ Chemical shifts from Köck et al. (ref. 2) 
Table S7. ROESY peaks assignments of the closed form of CycA in sample B-1 at $25^{\circ} \mathrm{C}$ that were used for MD calculations.

Res.1 Atom Res.2 Atom

Range $(\AA)$

! trans/cis peptide bonds ROEs

$\begin{array}{lllll}1 & \text { HA } & 2 & \text { HN } & 1.8-2.5 \\ 2 & \text { HA } & 3 & \text { NMe } & 1.8-2.5 \\ 3 & \text { HA1 } & 4 & \text { NMe } & 1.8-2.5 \\ 4 & \text { HA } & 5 & \text { HN } & 1.8-2.5 \\ 5 & \text { HA } & 6 & \text { NMe } & 1.8-2.5 \\ 6 & \text { HA } & 7 & \text { HN } & 1.8-2.5 \\ 7 & \text { HA } & 8 & \text { HN } & 1.8-2.5 \\ 8 & \text { HA } & 9 & \text { NMe } & 1.8-2.5 \\ 10 & \text { HA } & 11 & \text { NMe } & 1.8-2.5 \\ 11 & \text { HA } & 1 & \text { NMe } & 1.8-2.5\end{array}$

! cis peptide bonds ROES
9
$\mathrm{HA} \quad 10$
HA
$1.8-3.0$

! intra residue ROES

\begin{tabular}{|c|c|c|c|c|}
\hline 1 & $\mathrm{HA}$ & 1 & HD1\# & $1.8-5.0$ \\
\hline 1 & $\mathrm{HB}$ & 1 & HD1\# & $1.8-5.0$ \\
\hline 1 & $\mathrm{HB}$ & 1 & HG2 & $1.8-5.0$ \\
\hline 1 & HD1\# & 1 & HG2 & $1.8-5.0$ \\
\hline 1 & $\mathrm{HE}$ & 1 & HG2 & $1.8-5.0$ \\
\hline 1 & HG2 & 1 & NMe & $1.8-5.0$ \\
\hline 1 & NMe & 1 & $\mathrm{HA}$ & $1.8-5.0$ \\
\hline 1 & NMe & 1 & $\mathrm{HB}$ & $1.8-5.0$ \\
\hline 1 & $\mathrm{NMe}$ & 1 & HD1\# & $1.8-5.0$ \\
\hline 1 & $\mathrm{HZ}$ & 1 & $\mathrm{NMe}$ & $1.8-5.0$ \\
\hline 1 & HB & 1 & HD22 & $1.8-5.0$ \\
\hline 1 & HD1\# & 1 & HD22 & $1.8-5.0$ \\
\hline 1 & HD22 & 1 & $\mathrm{HA}$ & $1.8-5.0$ \\
\hline 1 & HD21 & 1 & $\mathrm{HB}$ & $1.8-5.0$ \\
\hline 1 & HD21 & 1 & $\mathrm{HZ}$ & $1.8-5.0$ \\
\hline 1 & HD21 & 1 & HG2 & $1.8-5.0$ \\
\hline 1 & $\mathrm{HE}$ & 1 & HD22 & $1.8-5.0$ \\
\hline 1 & NMe & 1 & HD22 & $1.8-5.0$ \\
\hline 2 & HG\# & 2 & $\mathrm{HA}$ & $1.8-5.0$ \\
\hline 2 & HG\# & 2 & $\mathrm{HN}$ & $1.8-5.0$ \\
\hline 2 & HB1 & 2 & $\mathrm{HN}$ & $1.8-5.0$ \\
\hline 2 & HB2 & 2 & $\mathrm{HN}$ & $1.8-5.0$ \\
\hline 4 & $\mathrm{HA}$ & 4 & NMe & $1.8-5.0$ \\
\hline 4 & NMe & 4 & $H G$ & $1.8-5.0$ \\
\hline 4 & $\mathrm{HA}$ & 4 & HD2\# & $1.8-5.0$ \\
\hline 4 & HD1\# & 4 & $\mathrm{HA}$ & $1.8-5.0$ \\
\hline 4 & HB2 & 4 & NMe & $1.8-5.0$ \\
\hline 4 & HD2\# & 4 & HB1 & $1.8-5.0$ \\
\hline 4 & HD1\# & 4 & HB1 & $1.8-5.0$ \\
\hline
\end{tabular}




\begin{tabular}{|c|c|c|c|c|}
\hline 4 & HD1\# & 4 & HB2 & $1.8-5.0$ \\
\hline 5 & $\mathrm{HA}$ & 5 & $\mathrm{HN}$ & $1.8-5.0$ \\
\hline 5 & $\mathrm{HB}$ & 5 & $\mathrm{HN}$ & $1.8-5.0$ \\
\hline 5 & HA & 5 & HG1\# & $1.8-5.0$ \\
\hline 5 & HA & 5 & HG2\# & $1.8-5.0$ \\
\hline 5 & HG2\# & 5 & HN & $1.8-5.0$ \\
\hline 6 & HA & 6 & $\mathrm{NMe}$ & $1.8-5.0$ \\
\hline 6 & NMe & 6 & HG & $1.8-5.0$ \\
\hline 6 & HD2\# & 6 & $\mathrm{HA}$ & $1.8-5.0$ \\
\hline 6 & HD1\# & 6 & $\mathrm{HA}$ & $1.8-5.0$ \\
\hline 6 & HD1\# & 6 & HB2 & $1.8-5.0$ \\
\hline 6 & HD2\# & 6 & HB1 & $1.8-5.0$ \\
\hline 6 & HD1\# & 6 & HB1 & $1.8-5.0$ \\
\hline 6 & NMe & 6 & HB1 & $1.8-5.0$ \\
\hline 6 & NMe & 6 & HB2 & $1.8-5.0$ \\
\hline 7 & $\mathrm{HN}$ & 7 & HA & $1.8-5.0$ \\
\hline 7 & $\mathrm{HB} \#$ & 7 & $\mathrm{HN}$ & $1.8-5.0$ \\
\hline 8 & HA & 8 & $\mathrm{HN}$ & $1.8-5.0$ \\
\hline 8 & $\mathrm{HB} \#$ & 8 & $\mathrm{HN}$ & $1.8-5.0$ \\
\hline 9 & HA & 9 & HG & $1.8-5.0$ \\
\hline 9 & NMe & 9 & HG & $1.8-5.0$ \\
\hline 9 & NMe & 9 & HB2 & $1.8-5.0$ \\
\hline 9 & HB1 & 9 & HD1\# & $1.8-5.0$ \\
\hline 9 & HB1 & 9 & HD2\# & $1.8-5.0$ \\
\hline 9 & HB2 & 9 & HD1\# & $1.8-5.0$ \\
\hline 9 & $\mathrm{HA}$ & 9 & HD2\# & $1.8-5.0$ \\
\hline 10 & HA & 10 & HG & $1.8-5.0$ \\
\hline 10 & $\mathrm{HA}$ & 10 & NMe & $1.8-5.0$ \\
\hline 10 & $\mathrm{NMe}$ & 10 & $\mathrm{HG}$ & $1.8-5.0$ \\
\hline 10 & HA & 10 & HD1\# & $1.8-5.0$ \\
\hline 10 & HA & 10 & HD2\# & $1.8-5.0$ \\
\hline 10 & HB1 & 10 & HD2\# & $1.8-5.0$ \\
\hline 10 & NMe & 10 & HB1 & $1.8-5.0$ \\
\hline 10 & NMe & 10 & HB2 & $1.8-5.0$ \\
\hline 11 & HB & 11 & NMe & $1.8-5.0$ \\
\hline 11 & $\mathrm{NMe}$ & 11 & HA & $1.8-5.0$ \\
\hline 11 & NMe & 11 & HG2\# & $1.8-5.0$ \\
\hline ! $\mathrm{N}-\mathrm{N}+1$ & sequent $i$ & & & \\
\hline 1 & HB & 2 & $\mathrm{HN}$ & $1.8-5.0$ \\
\hline 1 & NMe & 11 & HG1\# & $1.8-5.0$ \\
\hline 4 & NMe & 5 & $\mathrm{HN}$ & $1.8-5.0$ \\
\hline 4 & NMe & 5 & HG2\# & $1.8-5.0$ \\
\hline 5 & HB & 6 & NMe & $1.8-5.0$ \\
\hline
\end{tabular}




\begin{tabular}{|c|c|c|c|c|c|}
\hline & 5 & HG1\# & 6 & NMe & $1.8-5.0$ \\
\hline & 6 & HA & 7 & $\mathrm{HB} \#$ & $1.8-5.0$ \\
\hline & 6 & $\mathrm{NMe}$ & 7 & $\mathrm{HA}$ & $1.8-5.0$ \\
\hline & 8 & HB\# & 9 & HA & $1.8-5.0$ \\
\hline & 8 & $\mathrm{HB} \#$ & 9 & NMe & $1.8-5.0$ \\
\hline & 9 & HA & 10 & HG & $1.8-5.0$ \\
\hline & 9 & HA & 10 & HD1\# & $1.8-5.0$ \\
\hline & 9 & HA & 10 & HD2\# & $1.8-5.0$ \\
\hline & 10 & NMe & 11 & HA & $1.8-5.0$ \\
\hline $\mathrm{N}+$ & ROES & & & & \\
\hline & 1 & HB & 3 & $\mathrm{NMe}$ & $1.8-5.0$ \\
\hline & 1 & $\mathrm{HA}$ & 6 & HA & $1.8-5.0$ \\
\hline & 1 & HD1\# & 6 & $\mathrm{HA}$ & $1.8-5.0$ \\
\hline & 1 & HD1\# & 6 & HB2 & $1.8-5.0$ \\
\hline & 1 & $\mathrm{HZ}$ & 6 & HB2 & $1.8-5.0$ \\
\hline & 1 & HA & 7 & HB\# & $1.8-5.0$ \\
\hline & 1 & $\mathrm{HA}$ & 7 & HN & $1.8-5.0$ \\
\hline & 1 & HD1\# & 10 & HG & $1.8-5.0$ \\
\hline & 1 & $\mathrm{HA}$ & 11 & HG2\# & $1.8-5.0$ \\
\hline & 1 & HB & 11 & HG2\# & $1.8-5.0$ \\
\hline & 2 & HN & 5 & $H B$ & $1.8-5.0$ \\
\hline & 2 & HB1 & 5 & HB & $1.8-5.0$ \\
\hline & 2 & HB2 & 5 & $\mathrm{HB}$ & $1.8-5.0$ \\
\hline & 6 & HB1 & 10 & HD1\# & $1.8-5.0$ \\
\hline & 7 & HB\# & 11 & NMe & $1.8-5.0$ \\
\hline & 7 & $\mathrm{HN}$ & 11 & NMe & $1.8-5.0$ \\
\hline & 7 & HA & 11 & NMe & $1.8-5.0$ \\
\hline & 8 & HA & 11 & $\mathrm{NMe}$ & $1.8-5.0$ \\
\hline & 8 & $\mathrm{HN}$ & 11 & $\mathrm{NMe}$ & $1.8-5.0$ \\
\hline & 9 & HA & 11 & NMe & $1.8-5.0$ \\
\hline
\end{tabular}


Table S8. ROESY peaks assignments of the $\mathrm{CycA}: \mathrm{Ca}^{2+}$ in sample $\mathrm{B}-3$ at $5^{\circ} \mathrm{C}$ that were used for MD calculations.

\section{Res.1 Atom Res.2 Atom Range $(\AA)$}

! BACKBONE ALL TRANS

$\begin{array}{lllll}1 & \text { NMe } & 11 & \text { HA } & 2.0-3 . \odot \\ 1 & \text { HA } & 2 & \text { HN } & 2.0-3 . \odot \\ 2 & \text { HA } & 3 & \text { NMe } & 2.0-3.0 \\ 3 & \text { HA1 } & 4 & \text { NMe } & 2.0-3 . \odot \\ 3 & \text { HA2 } & 4 & \text { NMe } & 2.0-3 . \odot \\ 4 & \text { HA } & 5 & \text { HN } & 2.0-3 . \odot \\ 5 & \text { HA } & 6 & \text { NMe } & 2.0-3.0 \\ 6 & \text { HA } & 7 & \text { HN } & 2.0-3 . \odot \\ 7 & \text { HA } & 8 & \text { HN } & 2.0-3 . \odot \\ 8 & \text { HA } & 9 & \text { NMe } & 2.0-3 . \odot \\ 9 & \text { HA } & 10 & \text { NMe } & 2.0-3.0 \\ 10 & \text { HA } & 11 & \text { NMe } & 2.0-3 . \odot\end{array}$

\section{! INTRARESIDUES}

\begin{tabular}{|c|c|c|c|c|}
\hline 1 & HA & 1 & $H B$ & $1.8-5.0$ \\
\hline 1 & HA & 1 & HD1\# & $1.8-5.0$ \\
\hline 1 & $\mathrm{HA}$ & 1 & HG2 & $1.8-5.0$ \\
\hline 1 & HA & 1 & HG2 & $1.8-5.0$ \\
\hline 1 & HA & 1 & NMe & $1.8-5.0$ \\
\hline 1 & HB & 1 & HD1\# & $1.8-5.0$ \\
\hline 1 & HB & 1 & HD21 & $1.8-5.0$ \\
\hline 1 & HB & 1 & HD22 & $1.8-5.0$ \\
\hline 1 & HB & 1 & $\mathrm{HE}$ & $1.8-5.0$ \\
\hline 1 & HB & 1 & HG2 & $1.8-5.0$ \\
\hline 1 & HB & 1 & $\mathrm{NMe}$ & $1.8-5.0$ \\
\hline 1 & HD1\# & 1 & HD22 & $1.8-5.0$ \\
\hline 1 & HD1\# & 1 & HE & $1.8-5.0$ \\
\hline 1 & HD1\# & 1 & HG2 & $1.8-5.0$ \\
\hline 1 & HD1\# & 1 & $\mathrm{NMe}$ & $1.8-5.0$ \\
\hline 1 & HD21 & 1 & HD1\# & $1.8-5.0$ \\
\hline 1 & HD21 & 1 & $\mathrm{HZ}$ & $1.8-5.0$ \\
\hline 1 & HD22 & 1 & HE & $1.8-5.0$ \\
\hline 1 & HD22 & 1 & HG2 & $1.8-5.0$ \\
\hline 1 & HG2 & 1 & HE & $1.8-5.0$ \\
\hline 1 & HG2 & 1 & $\mathrm{NMe}$ & $1.8-5.0$ \\
\hline 1 & $\mathrm{HH} \#$ & 1 & $\mathrm{HZ}$ & $1.8-5.0$ \\
\hline 2 & HA & 2 & HB1 & $1.8-5.0$ \\
\hline 2 & HA & 2 & HG\# & $1.8-5.0$ \\
\hline 2 & HA & 2 & $\mathrm{HN}$ & $1.8-5.0$ \\
\hline 2 & HB1 & 2 & $\mathrm{HN}$ & $1.8-5.0$ \\
\hline 2 & HB2 & 2 & HA & $1.8-5.0$ \\
\hline 2 & HB2 & 2 & $\mathrm{HN}$ & $1.8-5.0$ \\
\hline 2 & HG\# & 2 & HB1 & $1.8-5.0$ \\
\hline 2 & HG\# & 2 & $\mathrm{HN}$ & $1.8-5.0$ \\
\hline 3 & HA2 & 3 & NMe & $1.8-5.0$ \\
\hline
\end{tabular}




\begin{tabular}{|c|c|c|c|c|}
\hline 4 & HA & 4 & HB1 & $1.8-5.0$ \\
\hline 4 & HA & 4 & HB2 & $1.8-5.0$ \\
\hline 4 & HA & 4 & HD1\# & $1.8-5.0$ \\
\hline 4 & HA & 4 & HD2\# & $1.8-5.0$ \\
\hline 4 & HA & 4 & HG & $1.8-5.0$ \\
\hline 4 & HA & 4 & $\mathrm{NMe}$ & $1.8-5.0$ \\
\hline 4 & HB1 & 4 & HD1\# & $1.8-5.0$ \\
\hline 4 & HB1 & 4 & HG & $1.8-5.0$ \\
\hline 4 & HB1 & 4 & $\mathrm{NMe}$ & $1.8-5.0$ \\
\hline 4 & HB2 & 4 & $\mathrm{NMe}$ & $1.8-5.0$ \\
\hline 4 & HD2\# & 4 & HB1 & $1.8-5.0$ \\
\hline 4 & HD2\# & 4 & HB2 & $1.8-5.0$ \\
\hline 4 & HG & 4 & $\mathrm{NMe}$ & $1.8-5.0$ \\
\hline 5 & $\mathrm{HA}$ & 5 & HG1\# & $1.8-5.0$ \\
\hline 5 & HA & 5 & HG2\# & $1.8-5.0$ \\
\hline 5 & HB & 5 & $\mathrm{HN}$ & $1.8-5.0$ \\
\hline 5 & HG1\# & 5 & $\mathrm{HN}$ & $1.8-5.0$ \\
\hline 5 & HG2\# & 5 & HB & $1.8-5.0$ \\
\hline 5 & $\mathrm{HN}$ & 5 & HA & $1.8-5.0$ \\
\hline 6 & HA & 6 & HD2\# & $1.8-5.0$ \\
\hline 6 & HA & 6 & HG & $1.8-5.0$ \\
\hline 6 & HA & 6 & $\mathrm{NMe}$ & $1.8-5.0$ \\
\hline 6 & HB1 & 6 & HA & $1.8-5.0$ \\
\hline 6 & HD1\# & 6 & $\mathrm{NMe}$ & $1.8-5.0$ \\
\hline 6 & HD2\# & 6 & HB2 & $1.8-5.0$ \\
\hline 6 & HD2\# & 6 & $\mathrm{NMe}$ & $1.8-5.0$ \\
\hline 6 & $H G$ & 6 & $\mathrm{NMe}$ & $1.8-5.0$ \\
\hline 7 & HA & 7 & HB\# & $1.8-5.0$ \\
\hline 7 & $\mathrm{HA}$ & 7 & $\mathrm{HN}$ & $1.8-5.0$ \\
\hline 7 & $\mathrm{HB} \#$ & 7 & $\mathrm{HN}$ & $1.8-5.0$ \\
\hline 8 & HA & 8 & HB\# & $1.8-5.0$ \\
\hline 8 & $\mathrm{HB} \#$ & 8 & $\mathrm{HN}$ & $1.8-5.0$ \\
\hline 9 & HA & 9 & HB2 & $1.8-5.0$ \\
\hline 9 & $\mathrm{HA}$ & 9 & HD1\# & $1.8-5.0$ \\
\hline 9 & $\mathrm{HA}$ & 9 & HD2\# & $1.8-5.0$ \\
\hline 9 & $\mathrm{HA}$ & 9 & HG & $1.8-5.0$ \\
\hline 9 & $\mathrm{HA}$ & 9 & $\mathrm{NMe}$ & $1.8-5.0$ \\
\hline 9 & HB1 & 9 & HA & $1.8-5.0$ \\
\hline 9 & HB2 & 9 & HD1\# & $1.8-5.0$ \\
\hline 9 & HB1 & 9 & HG & $1.8-5.0$ \\
\hline 9 & HB2 & 9 & $\mathrm{NMe}$ & $1.8-5.0$ \\
\hline 9 & HD1\# & 9 & $\mathrm{NMe}$ & $1.8-5.0$ \\
\hline 9 & HD2\# & 9 & $\mathrm{NMe}$ & $1.8-5.0$ \\
\hline 9 & HG & 9 & $\mathrm{NMe}$ & $1.8-5.0$ \\
\hline 10 & HA & 10 & HB2 & $1.8-5.0$ \\
\hline 10 & $\mathrm{HA}$ & 10 & HD2\# & $1.8-5.0$ \\
\hline 10 & HA & 10 & NMe & $1.8-5.0$ \\
\hline
\end{tabular}




\begin{tabular}{|c|c|c|c|c|}
\hline 10 & HB1 & 10 & HB2 & $1.8-5.0$ \\
\hline 10 & HB2 & 10 & $\mathrm{NMe}$ & $1.8-5.0$ \\
\hline 10 & HD1\# & 10 & HB2 & $1.8-5.0$ \\
\hline 10 & HG & 10 & $\mathrm{HA}$ & $1.8-5.0$ \\
\hline 11 & HA & 11 & $\mathrm{HB}$ & $1.8-5.0$ \\
\hline 11 & $\mathrm{HA}$ & 11 & HG1\# & $1.8-5.0$ \\
\hline 11 & HB & 11 & HG2\# & $1.8-5.0$ \\
\hline 11 & $\mathrm{HB}$ & 11 & NMe & $1.8-5.0$ \\
\hline 11 & HG1\# & 11 & HA & $1.8-5.0$ \\
\hline 11 & HG2\# & 11 & $\mathrm{NMe}$ & $1.8-5.0$ \\
\hline 11 & NMe & 11 & HA & $1.8-5.0$ \\
\hline ! $N-N+1$ & SEQUENTIAL & & & \\
\hline 1 & HB & 2 & $\mathrm{HN}$ & $1.8-5.0$ \\
\hline 1 & HB & 2 & HA & $1.8-5.0$ \\
\hline 1 & HB & 2 & HG\# & $1.8-5.0$ \\
\hline 2 & HB1 & 3 & NMe & $1.8-5.0$ \\
\hline 2 & HB2 & 3 & NMe & $1.8-5.0$ \\
\hline 2 & HG\# & 3 & NMe & $1.8-5.0$ \\
\hline 3 & HA2 & 4 & HA & $1.8-5.0$ \\
\hline 3 & NMe & 4 & HA & $1.8-5.0$ \\
\hline 4 & HD2\# & 5 & HN & $1.8-5.0$ \\
\hline 4 & NMe & 5 & HA & $1.8-5.0$ \\
\hline 4 & $\mathrm{NMe}$ & 5 & HG2\# & $1.8-5.0$ \\
\hline 5 & HG1\# & 6 & $\mathrm{NMe}$ & $1.8-5.0$ \\
\hline 5 & $\mathrm{HA}$ & 6 & HA & $1.8-5.0$ \\
\hline 5 & HG1\# & 6 & HA & $1.8-5.0$ \\
\hline 5 & $\mathrm{HN}$ & 6 & HD1\# & $1.8-5.0$ \\
\hline 5 & HG1\# & 6 & HD2\# & $1.8-5.0$ \\
\hline 6 & HB1 & 7 & HA & $1.8-5.0$ \\
\hline 6 & HD1\# & 7 & $\mathrm{HN}$ & $1.8-5.0$ \\
\hline 6 & HG & 7 & HA & $1.8-5.0$ \\
\hline 6 & $\mathrm{NMe}$ & 7 & HB\# & $1.8-5.0$ \\
\hline 6 & NMe & 7 & HN & $1.8-5.0$ \\
\hline 6 & HA & 7 & HB\# & $1.8-5.0$ \\
\hline 6 & HA & 7 & HA & $1.8-5.0$ \\
\hline 7 & HA & 8 & HB\# & $1.8-5.0$ \\
\hline 7 & HB\# & 8 & HA & $1.8-5.0$ \\
\hline 8 & HB\# & 9 & HD1\# & $1.8-5.0$ \\
\hline 8 & HB\# & 9 & NMe & $1.8-5.0$ \\
\hline 8 & HA & 9 & HB2 & $1.8-5.0$ \\
\hline 9 & HB2 & 10 & HD2\# & $1.8-5.0$ \\
\hline 9 & HB1 & 10 & $\mathrm{NMe}$ & $1.8-5.0$ \\
\hline
\end{tabular}




$\begin{array}{lllll}9 & \text { NMe } & 10 & \text { HA } & 1.8-5.0 \\ 9 & \text { NMe } & 10 & \text { HD2\# } & 1.8-5.0 \\ 9 & \text { HB1 } & 10 & \text { HB1 } & 1.8-5.0 \\ & & & & \\ 10 & \text { HB1 } & 11 & \text { NMe } & 1.8-5 . \odot \\ 10 & \text { HD2\# } & 11 & \text { NMe } & 1.8-5.0 \\ 10 & \text { NMe } & 11 & \text { HA } & 1.8-5.0 \\ 10 & \text { HA } & 11 & \text { HG2\# } & 1.8-5.0 \\ & & & & \\ 11 & \text { HG1\# } & 1 & \text { NMe } & 1.8-5.0 \\ 11 & \text { HG1\# } & 1 & \text { HB } & 1.8-5.0 \\ 11 & \text { HG2\# } & 1 & \text { HD22 } & 1.8-5.0 \\ 11 & \text { HG2\# } & 1 & \text { HE } & 1.8-5.0 \\ 11 & \text { NMe } & 1 & \text { HZ } & 1.8-5.0 \\ 11 & \text { NMe } & 1 & \text { HD1\# } & 1.8-5.0 \\ 11 & \text { NMe } & 1 & \text { HG2 } & 1.8-5.0 \\ 11 & \text { HG2\# } & 1 & \text { NMe } & 1.8-5.0\end{array}$

! N+ ROES

$\begin{array}{lllll}1 & \text { NMe } & 10 & \text { HA } & 1.8-5.0 \\ 1 & \text { HE } & 10 & \text { HD2\# } & 1.8-5.0 \\ 1 & \text { NMe } & 10 & \text { HB2 } & 1.8-5.0 \\ 1 & \text { HE } & 10 & \text { HB1 } & 1.8-5.0 \\ 1 & \text { NMe } & 10 & \text { HB1 } & 1.8-5.0 \\ 1 & \text { HD22 } & 10 & \text { HD1\# } & 1.8-5.0 \\ 1 & \text { HE } & 10 & \text { HD1\# } & 1.8-5.0 \\ & & & & \\ 3 & \text { HA1 } & 5 & \text { HG2\# } & 1.8-5.0 \\ & & & & \\ 8 & \text { HA } & 10 & \text { NMe } & 1.8-5.0 \\ 1 & \text { NMe } & 5 & \text { HB } & 1.8-5.0 \\ 1 & \text { HZ } & 5 & \text { HG1\# } & 1.8-5.0 \\ 1 & \text { HZ } & 5 & \text { HG2\# } & 1.8-5.0 \\ 1 & \text { HD22 } & 5 & \text { HG2\# } & 1.8-5.0 \\ 1 & \text { HD1\# } & 6 & \text { NMe } & 1.8-5.0 \\ & & & & \\ 1 & \text { NMe } & 6 & \text { HA } & 1.8-5.0 \\ 1 & \text { NMe } & 6 & \text { HD2 } & 1.8-5.0 \\ 1 & \text { NMe } & 6 & \text { HG } & 1.8-5.0 \\ & & & & \\ 6 & \text { HA } & 10 & \text { NMe } & 1.8-5.0 \\ 6 & \text { HD2\# } & 10 & \text { NMe } & 1.8-5.0 \\ 6 & \text { HA } & 10 & \text { HB2 } & 1.8-5.0\end{array}$




\section{S2. Supporting Figures}

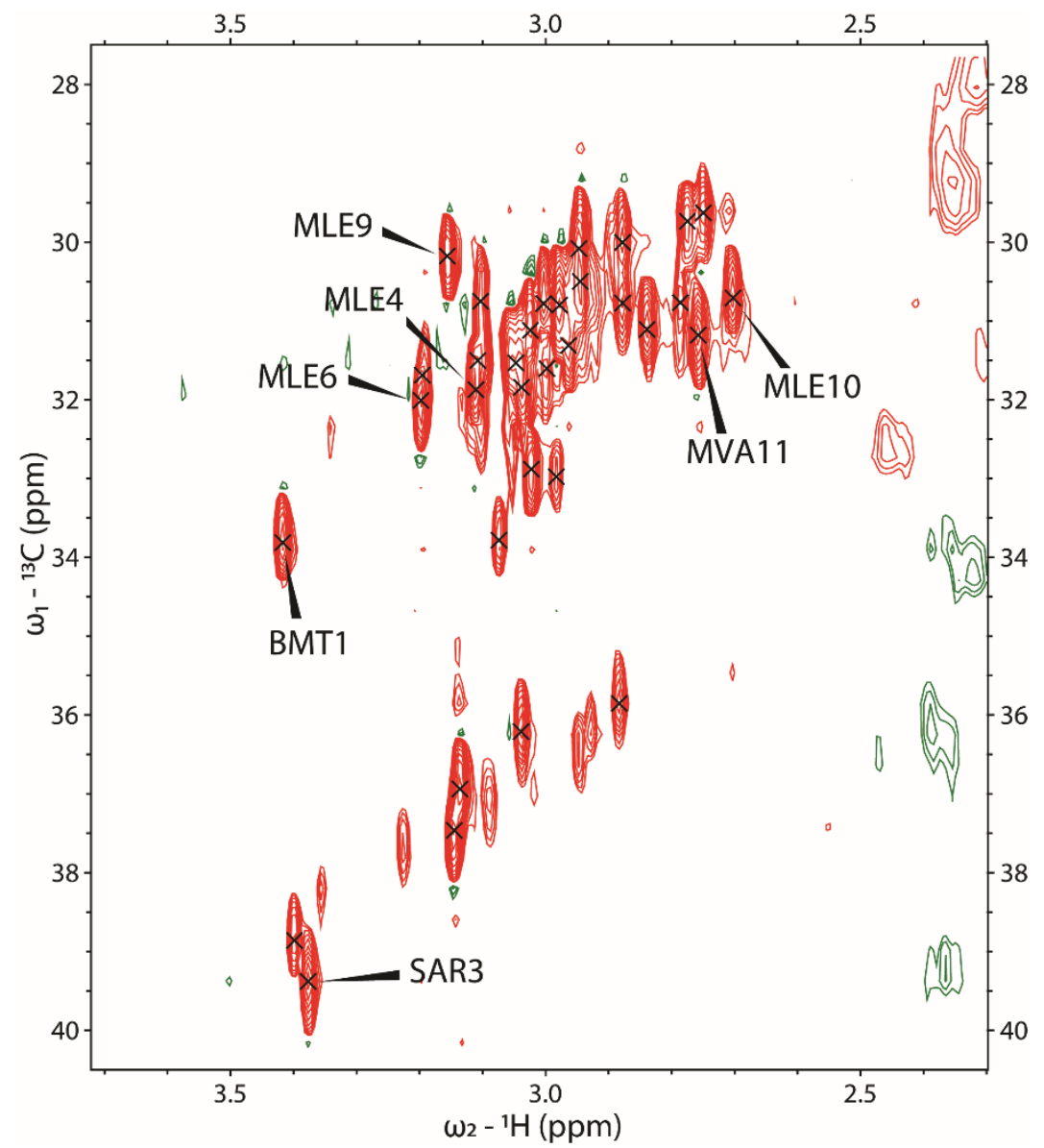

Figure S1. HSQC spectrum of free CycA in CD3OD:H2O/D2O (sample A-1).

Expanded region of the ${ }^{13} \mathrm{C}-\mathrm{HSQC}$ spectrum of CycA in CD3OD:H2O/D2O (sample A-1), showing the Nmethyl signals. The labelled signals correspond to the closed form of CycA. 


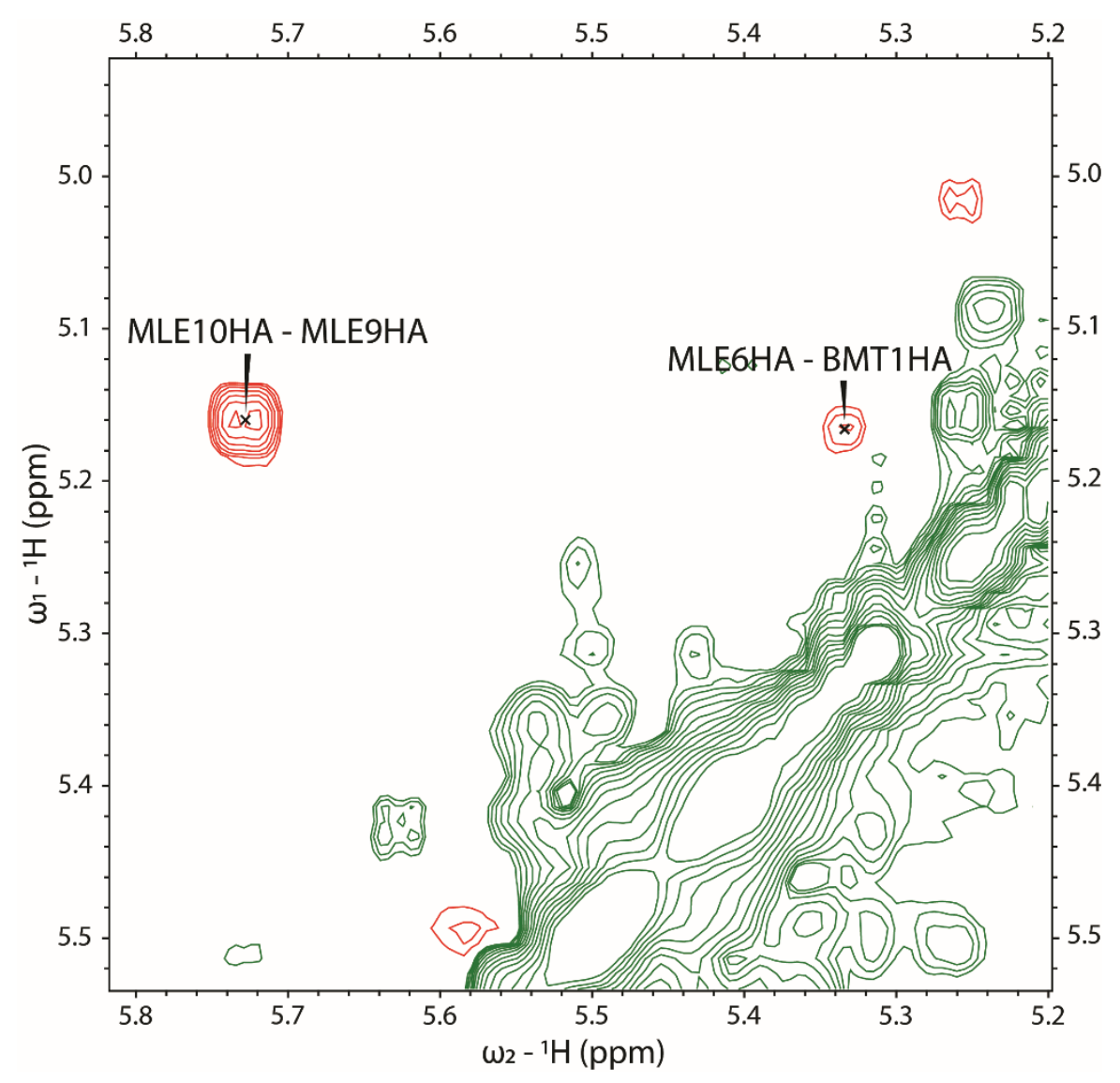

Figure S2. NOE signals showing characteristics of the closed form of CycA in CD3OD:H2O/D2O.

Expanded region of the ROESY spectrum of CycA in CD3OD:H2O/D2O (sample A1) at $15^{\circ} \mathrm{C}$, showing the $9 \mathrm{H} \alpha-10 \mathrm{H} \alpha$ and $1 \mathrm{H} \alpha-6 \mathrm{H} \alpha \mathrm{ROE}$ signals, characteristics of the closed form of CycA in solution. "MLE10HAMLE9HA", $9 \mathrm{H} \alpha-10 \mathrm{H} \alpha$ crosspeak indicates the cis peptide bond between MeLeu9 and MeLeu10.

"MLE6HA-BMT1HA", $1 \mathrm{H} \alpha-6 \mathrm{H} \alpha$ crosspeak indicates that MeBmt1 and MeLeu6 are close in space. In turn, this shows that the two antiparallel strands are close in space. 


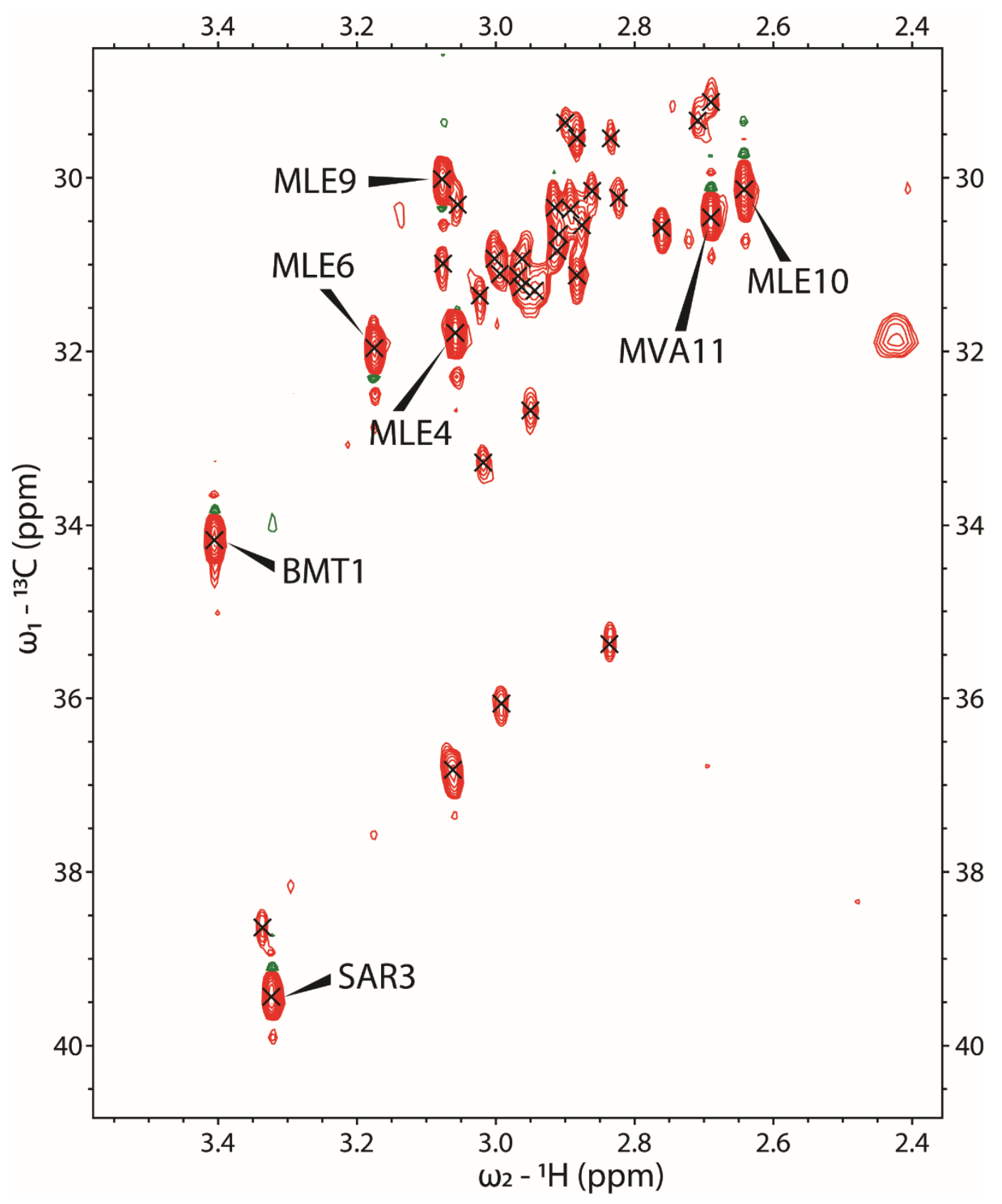

Figure S3. HSQC spectrum of free CyCA in CD3CN (sample B-1).

Expanded region of the ${ }^{13} \mathrm{C}-\mathrm{HSQC}$ spectrum of $\mathrm{CyCA}$ in $\mathrm{CD}_{3} \mathrm{CN}$ (sample $\mathrm{B}-1$ ), showing the N-methyl signals. The signals labelled correspond to the closed form of CycA. 


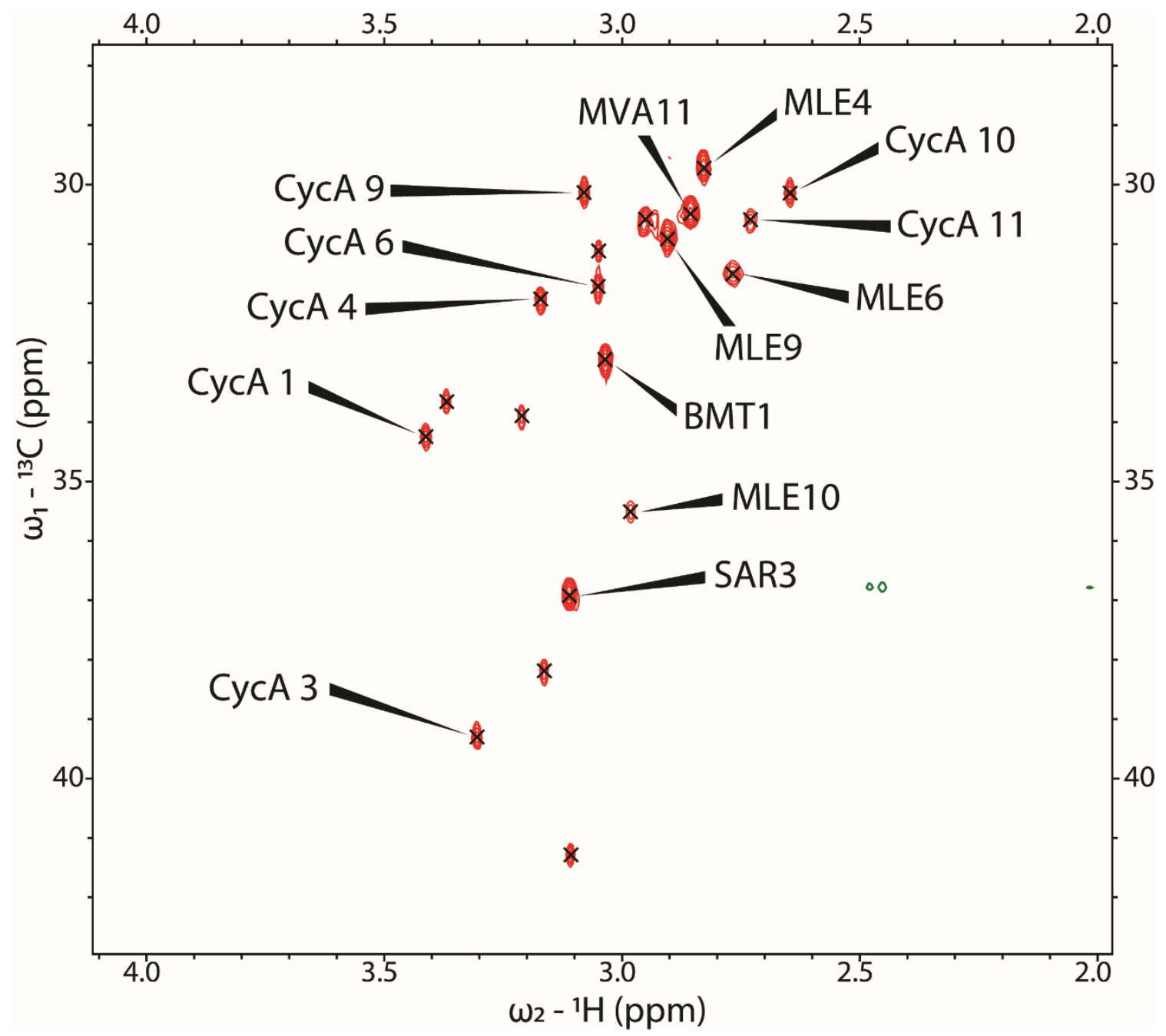

Figure S4. HSQC spectrum of CyCA:Ca ${ }^{2+}$ in $\mathrm{CD} 3 \mathrm{CN}$ (sample B-3).

Expanded region of the ${ }^{13} \mathrm{C}-\mathrm{HSQC}$ spectrum of $\mathrm{CycA}$ in $\mathrm{CD}_{3} \mathrm{CN}$ (sample $\mathrm{B}-3$ ) at $5{ }^{\circ} \mathrm{C}$, showing the $\mathrm{N}$-methyl signals. The signals labelled with the residue names correspond to $\mathrm{CycA}: \mathrm{Ca}^{2+}$. Those labelled with the prefix "CycA" correspond to the closed form of CycA. 


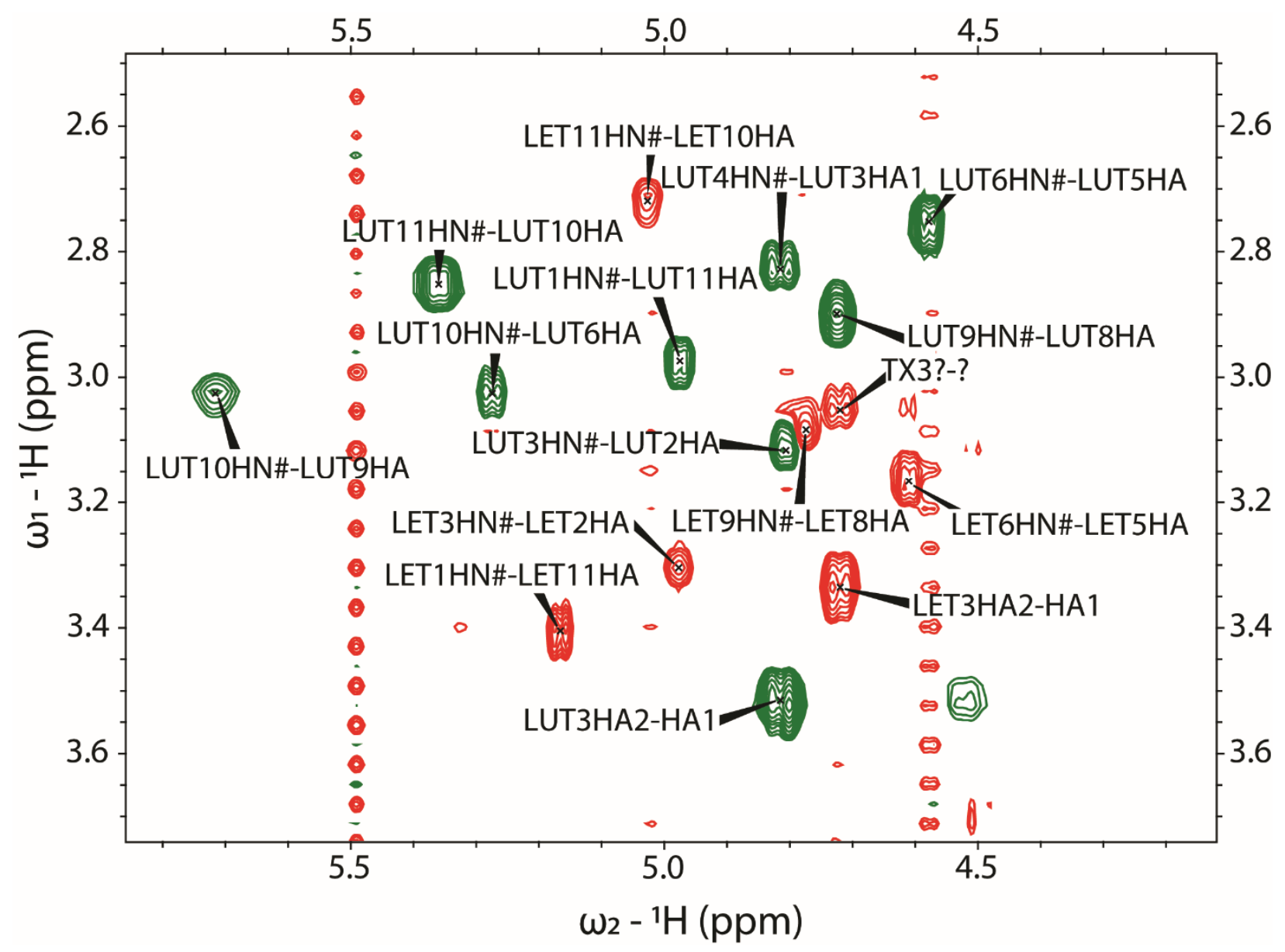

Figure S5. NOESY crosspeaks showing the three main conformations in sample B-3.

Expanded region of the NOESY spectrum of sample B-3 at $20^{\circ} \mathrm{C}$ [describe sample B-3 fully], showing the different phases of the NOEs for the different conformers. Positive NOEs for CyCA (LET) and negative for CycA $+\mathrm{Ca}^{2+}$ (LUT). Also, the peak labelled TX3?? from the $3^{\text {rd }}$ conformation of CycA in sample B3 has a positive phase. 

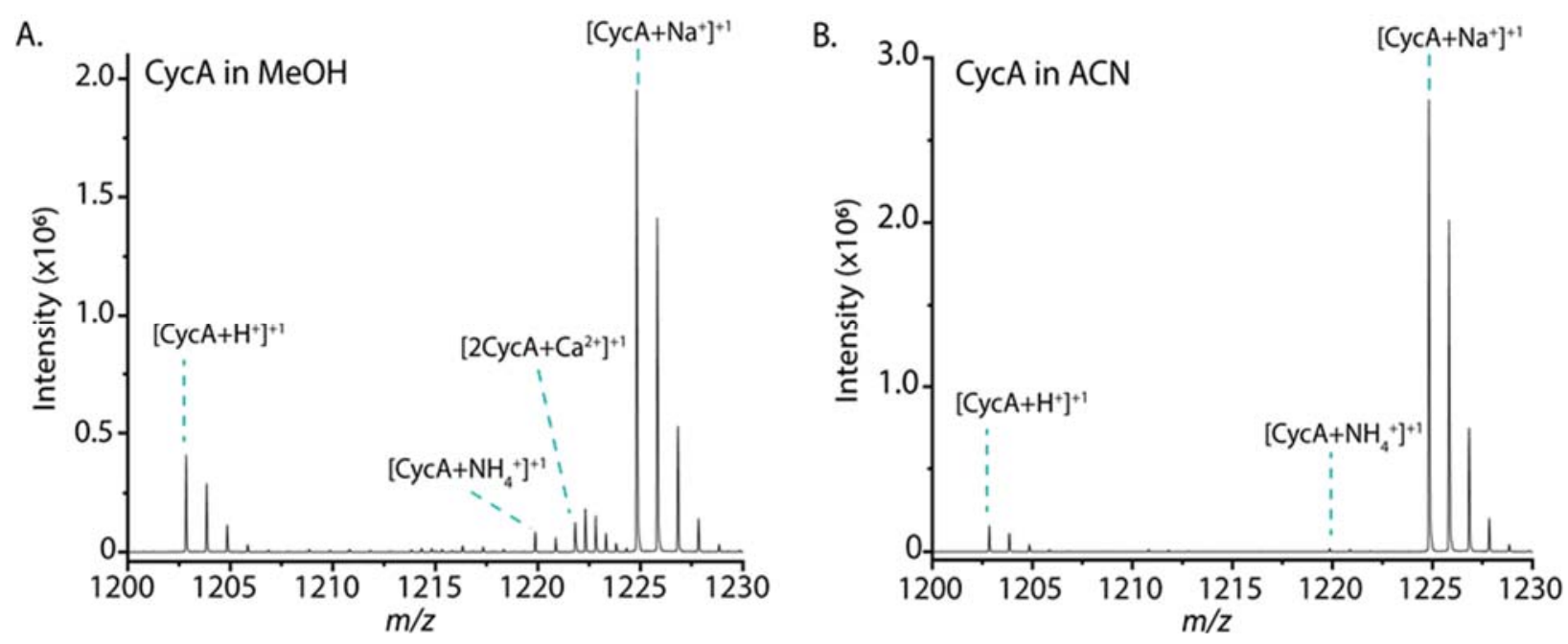

Figure S6. Mass spectra of CycA in methanol and acetonitrile.

The mass spectra of CycA shown in (A) methanol and (B) acetonitrile. The major difference is the lack of the calciated species when acetonitrile is used as the running buffer. 


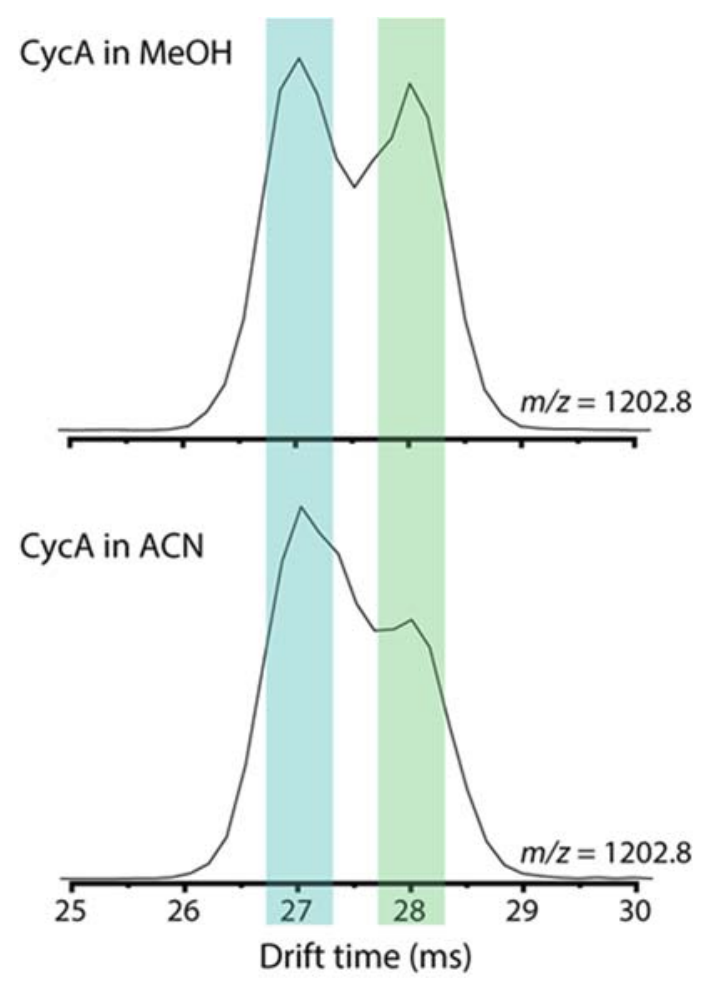

Figure S7. Arrival time distribution spectra of CycA in methanol and acetonitrile.

ATD of CycA in methanol (top panel) and acetonitrile (bottom panel). As solvent polarity decreases, the conformations are shifted towards the more compact structure (highlighted in blue). 


\section{References Cited}

(1) Kessler, H.; Köck, M.; Wein, T.; Gehrke, M. Reinvestigation of the Conformation of Cyclosporin A in Chloroform. Helv. Chim. Acta 1990, 73, 1818-1832.

(2) Köck, M.; Kessler, H.; Seebach, D.; Thaler, A. Novel Backbone Conformation of Cyclosporin A: The Complex with Lithium Chloride. J. Am. Chem. Soc. 1992, 114, 26762686. 